\title{
Quantitative detection of Streptococcus pneumoniae cells harbouring single or multiple copies of the gene encoding the green fluorescent protein
}

\author{
Paloma Acebo, Concha Nieto, María Angeles Corrales, Manuel Espinosa \\ and Paloma López
}

Author for correspondence: Manuel Espinosa. Tel: +34 915611800. Fax: +34915627518. e-mail:mespinosa@cib.csic.es

Centro de Investigaciones Biológicas, CSIC, Velázquez, 144, E-28006 Madrid, Spain

\begin{abstract}
A modified gfp gene from Aequorea victoria, encoding a variant of the green fluorescent protein (GFP), was subcloned into the mobilizable plasmid pMV158. gfp was placed under the control of the inducible $P_{\mathrm{M}}$ promoter of the Streptococcus pneumoniae gene maIM, cloned in plasmid pLS70. The $P_{\mathrm{M}}$ promoter is regulated by the product of the pneumococcal malR gene, which is inactivated by growing the cells in maltose-containing media. By homologous recombination, the $P_{\mathrm{m}}-\mathbf{g f p}$ construction was integrated into the host chromosome in a single copy. In both conditions (single and multiple copies), the pneumococcal cells were able to express GFP in an inducible or constitutive form, depending on whether the $S$. pneumoniae strain harboured a wild-type or a mutant malR gene. Quantification of the levels of GFP expressed by cultures supplemented with sucrose or maltose as carbon sources was feasible by fluorescence spectroscopy. Phase-contrast and fluorescence microscopy allowed pneumococcal cells expressing GFP in mixed cultures to be distinguished from those not carrying the gfp gene.
\end{abstract}

Keywords: green fluorescent protein, pneumococci, chromosomal integration, maltose regulon, MalR repressor

\section{INTRODUCTION}

One of the main problems in the identification of bacteria and assessment of their colonizing ability is the requirement for tedious biochemical and microbiological analyses. Some techniques have proven useful for these purposes, such as 'in situ' PCR and the use of bacteria tagged with the green fluorescent protein (GFP). In general, routine employment of GFP as a reporter has been limited to the detection of some Gram-negative bacteria, such as Escherichia coli and Pseudomonas syringae (Christensen et al., 1996; Miller \& Lindow, 1997). Among Gram-positive bacteria, the potential use of GFP as a reporter has also been documented (Geoffroy et al., 2000; Scott et al., 2000; Somkuti \& Steinberg, 1999), although it has only been in Bacillus subtilis that GFP has been used to follow more complex processes

Abbreviations: $\mathrm{Ap}$, ampicillin; $\mathrm{Cm}$, chloramphenicol; GFP, green fluorescent protein; Tc, tetracycline; TIR, translation initiation region. such as DNA replication (Lemon \& Grossman, 1998) and sporulation (Lewis \& Errington, 1996). Measurement of GFP activity requires post-translational oxidation of the protein, and full activity is only achieved at $\mathrm{pH}$ values around $7 \cdot 0$ (reviewed by Sullivan \& Kay, 1999). These features, although reversible, have hindered employment of this useful tool in some microaerophiles, such as the human pathogen Streptococcus pneumoniae.

We have been interested in the study of the utilization of maltosaccharides by $S$. pneumoniae (Puyet \& Espinosa, 1993; Stassi et al., 1982). Genes involved in this process are organized as a regulon composed of three operons. Two of them are involved in maltosaccharide uptake (malXCD) and its utilization (malMP), and one (malAR) is involved in regulation (Puyet \& Espinosa, 1993; Puyet et al., 1993). The two former operons are transcribed from two divergent promoters, termed $P_{\mathrm{M}}$ (for the malMP operon) and $P_{\mathrm{x}}$ (for the malXCD operon), which are negatively regulated by the product of malR 


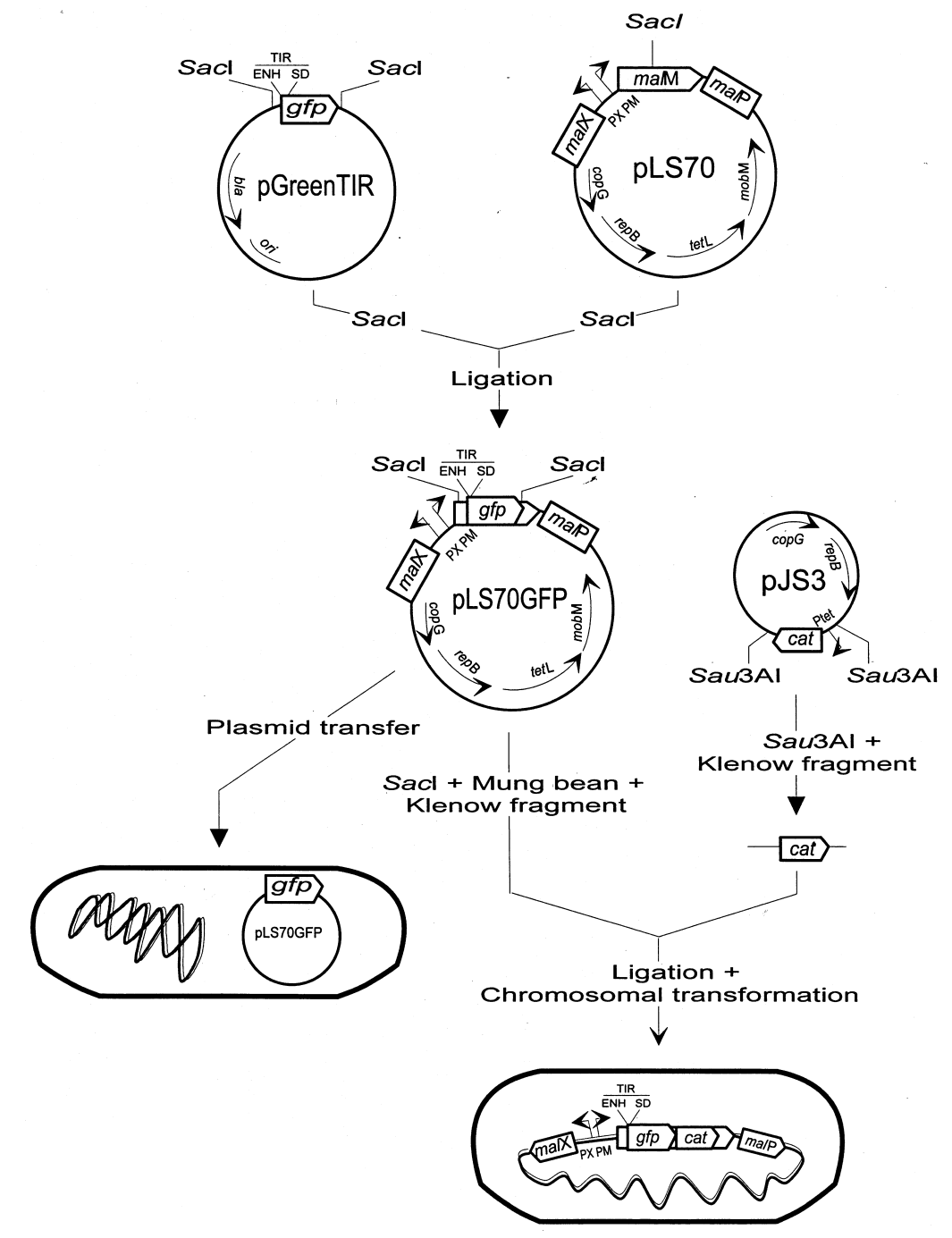

Fig. 1. Construction of strains harbouring the gfp gene. Plasmid pLS70GFP has the pMV158 replicon and carries the gfp gene placed under the control of promoter $P_{\mathrm{M}}$ (inducible by maltose) interrupting the malM gene, whereas promoter $P_{\mathrm{X}}$ directs synthesis of a truncated malX gene. Genes are indicated by arrows, with arrowheads pointing in the direction of transcription. Genes or DNA regions shown are those involved in pMV158 replication (repB, copG); antibiotic resistance [ampicillin (bla), chloramphenicol (cat), tetracycline (tetL)]; mobilization (mobM); fluorescence ( $g f p)$; and replication of pGreenTIR (ori). The translation initiation region (TIR), required to enhance translation of gfp, which contains an enhancer of translation (ENH) and a Shine-Dalgarno (SD) sequence, is also indicated. Promoters $P_{\mathrm{M}}(\mathrm{PM}), P_{\mathrm{X}}(\mathrm{PX})$ and $P_{\text {tet }}$ (Ptet) direct the transcription of malM, malX, and tet $L$ and cat, respectively.

(Nieto et al., 1997). MalR binds specifically to two operator sequences located in the intergenic region between operons malXCD and malMP (Nieto et al., 1997). The binding of MalR to its DNA target is reversed by the addition of maltose, pointing to inactivation of the repressor by the sugar (Nieto et al., 1997). In addition, the strong promoter $P_{\mathrm{M}}$ has proved to support functional constitutive expression of $g f p$ in Lactococcus lactis (Fernández de Palencia et al., 2000).

In this work, we used the recombinant plasmid pLS70 (Stassi et al., 1982), which harbours the pMV158 replicon (del Solar et al., 1998) and part of the pneumococcal mal regulon to clone a modified $g f p$ gene (Miller \& Lindow, 1997) from Aequorea victoria. In addition, by homologous recombination, we introduced the $g f p$ gene into a single chromosomal copy. Expression of GFP was achieved in S. pneumoniae in both genetic conditions (multiple or single copies), as detected by direct measurement of the fluorescence of the cells. A combination of phase-contrast and fluorescence microscopy allowed us to distinguish between cells harbouring or not harbouring the $g f p$ gene. Our results constitute a step forward in following directly the ability of pneumococcal cells to colonize epithelial cells.

\section{METHODS}

Bacterial strains and plasmids. All constructions were done in S. pneumoniae Rc19, a pneumococcal derivative strain synthesizing the MalM (amylomaltase) protein in a constitutive manner (Lacks, 1968). This strain, and S. pneumoniae R61 (Lacks, 1968), were also employed for expression and detection of GFP activity. Determination of the nucleotide sequence of the malR region of strain Rc19 showed that it harbours a deletion that removes four amino acids from the $\mathrm{N}$-terminal region of MalR. This deletion is included within a region supposedly involved in the binding of MalR to its DNA target, leading to inactivation of MalR (C. Nieto, unpublished). The plasmid used was pLS70, a pMV158 derivative (Burdett, 1980) in which a fragment of the pneumococcal mal regulon was cloned (Stassi et al., 1982). The cloned region contains promoters $P_{\mathrm{M}}$ and $P_{\mathrm{x}}$, the entire malM, and truncated malP and malX genes (Fig. 1). In addition, we used plasmid pGreenTIR (Miller \& Lindow, 1997), a derivative of pUC18 in 
which a mutated $g f p$ gene (containing a double F64L S65T amino acid change that increases GFP solubility and fluorescence) has been cloned. This plasmid also contains an improved translation initiation region (TIR) for prokaryotes, including the translational enhancer $(\mathrm{ENH})$ and the ShineDalgarno (SD) regions of phage T7 gene 10, so that synthesis of GFP is enhanced. Finally, plasmid pJS3 (Ballester et al., 1990) is a pMV158-based replicon in which the cat gene, encoding resistance to chloramphenicol $(\mathrm{Cm})$ of the staphylococcal plasmid pC194 (Horinouchi \& Weisblum, 1982) has been placed under the control of the promoter for the tet gene of pMV158. E. coli DH5 $\alpha$ cells were grown in TY medium (Sambrook et al., 1989), with selection for ampicillin (Ap) resistance $\left(100 \mu \mathrm{g} \mathrm{ml}^{-1}\right)$ for E. coli/pGreenTIR. S. pneumoniae cells were grown in AGCH medium (which contains casein hydrolysate, amino acids, vitamins, salts, albumin and catalase; Lacks, 1968), supplemented with sucrose, maltose or both sugars at a final concentration of $0 \cdot 8 \%$. To test whether $g f p$ expression was limited to this medium, cells were also grown in Todd-Hewitt Broth (Difco), supplemented with $0.5 \%$ yeast extract and $0.8 \%$ sugars as above. Selection was applied for resistance to tetracycline $(\mathrm{Tc})\left(1 \mu \mathrm{g} \mathrm{ml} \mathrm{m}^{-1}\right)$ or $\mathrm{Cm}$ $\left(5 \mu \mathrm{g} \mathrm{ml}^{-1}\right)$.

Plasmid DNA preparation and DNA manipulations. Pneumococcal cells were grown and transformed with plasmid DNA as described previously (Lacks et al., 1986). Purified plasmid DNA from $S$. pneumoniae was prepared as reported by del Solar et al. (1987). Restriction endonucleases, mung bean nuclease, the Klenow fragment of DNA polymerase I (PolIK) and DNA ligase were purchased from New England Biolabs or Boehringer Mannheim and were used as specified by the suppliers. Restriction fragments employed for cloning experiments were purified from agarose gels by phenol extraction (Sambrook et al., 1989). Determination of the nucleotide sequence of the desired DNA fragments of Rc19 and Rc19malM:: gfp cat was done after PCR amplification by the dideoxynucleotide termination procedure on an automated sequencer (ABI 373A).

Measurement of GFP activity. Pneumococcal cells harbouring plasmids or chromosomal inserts were grown in sugarcontaining media, without selective pressure, to an $\mathrm{OD}_{650}$ of 0.7 (about $6 \times 10^{8}$ c.f.u. $\mathrm{ml}^{-1}$ ). In some cases, fluorescence was directly measured in the culture medium. In other cases, cells ( $1.5 \mathrm{ml}$ from each culture) were sedimented by centrifugation and suspended in the same volume of PBS buffer $(10 \mathrm{mM}$ $\mathrm{Na}_{2} \mathrm{HPO}_{4}, 1 \mathrm{mM} \mathrm{KH_{2 }} \mathrm{PO}_{4}, 140 \mathrm{mM} \mathrm{NaCl}, 3 \mathrm{mM} \mathrm{KCl}, \mathrm{pH}$ $7 \cdot 2)$ prior to determination of their fluorescence. Cell lysates were prepared by sedimentation of the cells $(1.5 \mathrm{ml})$, suspension in the same volume of lysis buffer $(50 \mathrm{mM}$ Tris $/ \mathrm{HCl}$ pH 8.0, $10 \mathrm{mM}$ EDTA, $0 \cdot 1 \%$ sodium deoxycholate, $1 \%$ glucose) and incubation at $37^{\circ} \mathrm{C}$ for $10 \mathrm{~min}$. Aliquots $(200 \mu \mathrm{l})$ of cells or cell lysates were used to measure the fluorescence on a LS-50B spectrophotometer (Perkin-Elmer) by excitation at a wavelength of $488 \mathrm{~nm}$ with a slit width of 2.5 or $5 \mathrm{~nm}$ for cuvettes or microtitre plates, respectively, and detection of emission at $510 \mathrm{~nm}$ with a slit width of 10 or $5 \mathrm{~nm}$ for cuvettes or microtitre plates, respectively. As a control to determine the background fluorescence, isogenic strains (plasmid-free or carrying pLS70) were used. Analysis of the culture media and of supernatants of cultures grown to different optical densities showed that their intrinsic fluorescence decreased as a function of cell growth (not shown), indicating that fluorescent compounds in the media were consumed by the cells.

Fluorescence microscopy. Cultures of S. pneumoniae strains were grown in medium supplemented with sucrose plus maltose $(0 \cdot 8 \%$ each $)$ to an $\mathrm{OD}_{650}$ of $0 \cdot 4$. Cells in growth medium were directly analysed by phase-contrast or epifluorescence microscopy with a Zeiss Axioplan Universal microscope using excitation standard FITC set D480/30 and emission TBP 460/530/610 filters.

\section{RESULTS AND DISCUSSION}

\section{Construction of pLS70GFP and integration of $g f p$ gene into the pneumococcal chromosome}

To clone the gfp gene into plasmid pLS70, DNA from plasmid pGreenTIR was digested with $\operatorname{SacI}$ and the $0.8 \mathrm{~kb}$ DNA fragment, containing the $g f p$ gene with its own TIR signals, was purified. This fragment was ligated to DNA from pLS70 linearized at the single SacI site, located within the coding sequence of the malM gene (Fig. 1). The ligation mixture was used to transform competent pneumococcal cells, selecting for Tc resistance. Due to the high intrinsic fluorescence of the pneumococcal medium, direct selection for $\mathrm{Tc}^{\mathrm{R}}$ and fluorescent colonies was not feasible on agar plates. Plasmid DNA from several clones was prepared and plasmids of an appropriate size were selected and analysed by restriction mapping. The resulting plasmid was termed pLS70GFP, and the integrity of the $g f p$ gene cassette was checked by determination of its entire nucleotide sequence. This construction places the $g f p$ gene under the control of promoter $P_{\mathrm{M}}$ (Fig. 1).

To obtain a single copy of $g f p$, we used the procedure developed for $S$. pneumoniae based on recombination between the chromosomal DNA fragment present in pLS70GFP and the homologous chromosomal region in the host (López et al., 1982). As a selectable marker, we used the cat gene of plasmid pJS3, which is readily expressed in pneumococci because of the coupling of the tet promoter $\left(P_{\text {tet }}\right)$ of plasmid pMV158 to the cat gene of the staphylococcal plasmid pC194 (Ballester et al., 1989). Plasmid pLS70GFP was partially digested with SacI, the linear forms were purified and the protruding ends were made blunt by treatment with mung bean nuclease and filling in with PolIK. Likewise, DNA from plasmid pJS3 was cleaved with Sau3AI and the $1 \cdot 1 \mathrm{~kb}$ fragment containing the cat gene was purified and treated with PolIK. Both DNA fragments were ligated to generate $g f p-c a t$ or cat-gfp cassettes flanked by pneumococcal mal regions and the mixture was used to transform competent S. pneumoniae Rc19 cells. Since the donor DNA is taken up as linear single-stranded molecules by $S$. pneumoniae, chromosomal transformation is enhanced over plasmid establishment by a factor of 1000 when selection is applied for $\mathrm{Cm}$ resistance (López et al., 1982). Six transformants were selected and analysed for plasmid content and levels of fluorescence. None of them harboured plasmids, and three of them exhibited higher fluorescence than the parental strain (not shown). The chromosomal structure at the mal regulon of one of the fluorescent clones was checked by PCR amplification and determination of the nucleotide sequence. This clone, containing the desired construction with the insertion of the $g f p-c a t$ cassette within the malM gene (Fig. 1) was chosen and termed Rc19malM::gfp cat. 
Table 1. Fluorescence of pneumococcal cells in AGCH medium and in PBS buffer, and of cell lysates

Bacterial cultures were grown to an $\mathrm{OD}_{650}$ of $0 \cdot 4$ (about $4 \times 10^{8}$ c.f.u. $\mathrm{ml}^{-1}$ ) in $\mathrm{AGCH}$ plus sucrose, and total fluorescence of the cell lysates and of the living cells in the growth medium or after resuspension in PBS buffer was determined in microtitre plates. The mean \pm SD of three different cultures of each strain is shown. Corrected intensity corresponds to the fluorescence due to GFP and was calculated from the total fluorescence by subtraction of the values obtained in the corresponding isogenic strain lacking $g f p$ (either Rc19 or Rc19/pLS70).

\begin{tabular}{|c|c|c|c|c|c|c|c|}
\hline \multirow[t]{2}{*}{ Strain } & \multirow[t]{2}{*}{ Plasmid } & \multicolumn{2}{|c|}{$\mathrm{AGCH}$} & \multicolumn{2}{|c|}{ PBS } & \multicolumn{2}{|c|}{ Cell lysates } \\
\hline & & Intensity & $\begin{array}{c}\text { Corrected } \\
\text { intensity }\end{array}$ & Intensity & $\begin{array}{c}\text { Corrected } \\
\text { intensity }\end{array}$ & Intensity & $\begin{array}{c}\text { Corrected } \\
\text { intensity }\end{array}$ \\
\hline $\begin{array}{l}\text { S. pneumoniae Rc19 } \\
\text { malM::gfp cat }\end{array}$ & - & $52 \cdot 26 \pm 1 \cdot 48$ & $17 \cdot 08$ & $49 \cdot 44 \pm 2 \cdot 52$ & $28 \cdot 92$ & $57 \cdot 62 \pm 1 \cdot 2$ & $36 \cdot 06$ \\
\hline S. pneumoniae Rc19 & pLS70GFP & $154 \cdot 6 \pm 7 \cdot 74$ & $120 \cdot 34$ & $212 \cdot 78 \pm 6 \cdot 55$ & $192 \cdot 71$ & $277 \cdot 16 \pm 3 \cdot 5$ & $256 \cdot 07$ \\
\hline
\end{tabular}

\section{Expression of the gfp gene in S. pneumoniae}

Detection of GFP depends upon the $\mathrm{pH}$ of the medium and the levels of oxygen (Sullivan \& Kay, 1999). These features are reversible, but the use of GFP as a reporter in microaerophilic bacteria (such as pneumococci) usually demands exposure of cells to aerobic conditions, and neutralization and removal of fluorescent components of the culture media. These factors make it difficult to monitor the fluorescence of $S$. pneumoniae during cell growth. To test whether pneumococcal cells harbouring the $g f p$ gene expressed GFP, we analysed the fluorescence in intact cells (either in the culture medium or after washing the cells and resuspension in PBS buffer) and in cell lysates. The observed total fluorescence was corrected for the intrinsic fluorescence of cells or lysates detected in the isogenic strains lacking $g f p$. The results (Table 1 ) showed that expression of GFP could be measured, although the levels were about twofold higher when the cells were suspended in PBS buffer as compared with the values obtained in growth medium. Similar levels were found in cell lysates and in cells suspended in PBS. The intensity of fluorescence measured in S. pneumoniae/pLS70GFP was fairly high and reproducible, being about 6-7 times higher than those obtained for the strain Rc19malM::gfp cat. These differences are consistent with the levels of amylomaltase (encoded by the malM gene) found in $S$. pneumoniae/pLS70 compared with those synthesized by a single chromosomal copy of malM (Stassi et al., 1982). Similar levels of $g f p$ synthesis were detected when cells were grown in media other than AGCH (not shown). The above results allowed us to conclude that: (i) the $g f p$ gene from plasmid pGreenTIR is expressed in S. pneumoniae; (ii) GFP synthesized is fully active in living pneumococcal cells; (iii) GFP can be detected in culture media, even in cells harbouring a single copy of the gene; and (iv) the levels of GFP are related to the gene dosage.

We next tested whether: (i) the presence of the recombinant plasmids or the chromosomal construction had an adverse influence on cell growth, and (ii) a correlation between biomass and GFP expression existed. To this end, pneumococcal Rc19 cells were grown in medium containing sucrose plus maltose, and optical density (Fig. 2a) and fluorescence (Fig. 2b) of the cultures was monitored in the growth medium. The results showed that cell growth was slightly reduced when Rc19 cells harboured plasmids (pLS70 or pLS70GFP). The calculated doubling times were $40 \mathrm{~min}$ for plasmid-free cells and $45 \mathrm{~min}$ for plasmid-containing cells. However, strains harbouring $g f p$ were not impaired in growth (Fig. $2 a)$, they showed higher fluorescence than their isogenic strains lacking the $g f p$ gene, and only the fluorescence of $g f p^{+}$strains increased with cell mass (not shown), indicating that the fluorescence is correlated with cell growth. Corrected fluorescence values for Rc19/ pLS70GFP and Rc19malM::gfp cat strains (Fig. 2b) showed that, over the range of growth analysed, GFP expression increased linearly with cell mass and was related to gene dosage. Thus, the observed decrease in $\mathrm{pH}$ of the medium from 7.9 to 6.4 during growth of Rc19/pLS70 did not affect detection of GFP (top of Fig. $2 \mathrm{~b}$ ). In addition, alteration of the $\mathrm{pH}$ by addition of $\mathrm{NaOH}$ or $\mathrm{HCl}$ to the cultures revealed that full activity of GFP can be monitored within a $\mathrm{pH}$ range of $9 \cdot 5-6.0$ (not shown). We concluded that quantification of GFP in vivo can be achieved in a reliable manner during growth of $S$. pneumoniae in medium down to $\mathrm{pH} 6 \cdot 0$. Our results also showed that GFP can be used to monitor pneumococcal cells in real time.

\section{Constitutive and inducible expression of $g f p$ in pneumococcal cells}

Promoter $P_{\mathrm{M}}$ is inducible by maltose in $S$. pneumoniae, being regulated by the MalR repressor (Nieto et al., 1997). This would not be the case for strain Rc19 lacking a functional MalR, in which expression of $g f p$ from $P_{M}$ should be constitutive and independent of the carbon source. To compare the levels of GFP synthesized in strain Rc19 with those in a wild-type background (i.e. one intact copy of the malR gene), both the chromosomal $g f p-c a t$ gene cassette and plasmid pLS70GFP were independently transferred by transformation into the wild-type S. pneumoniae R61. After checking the 

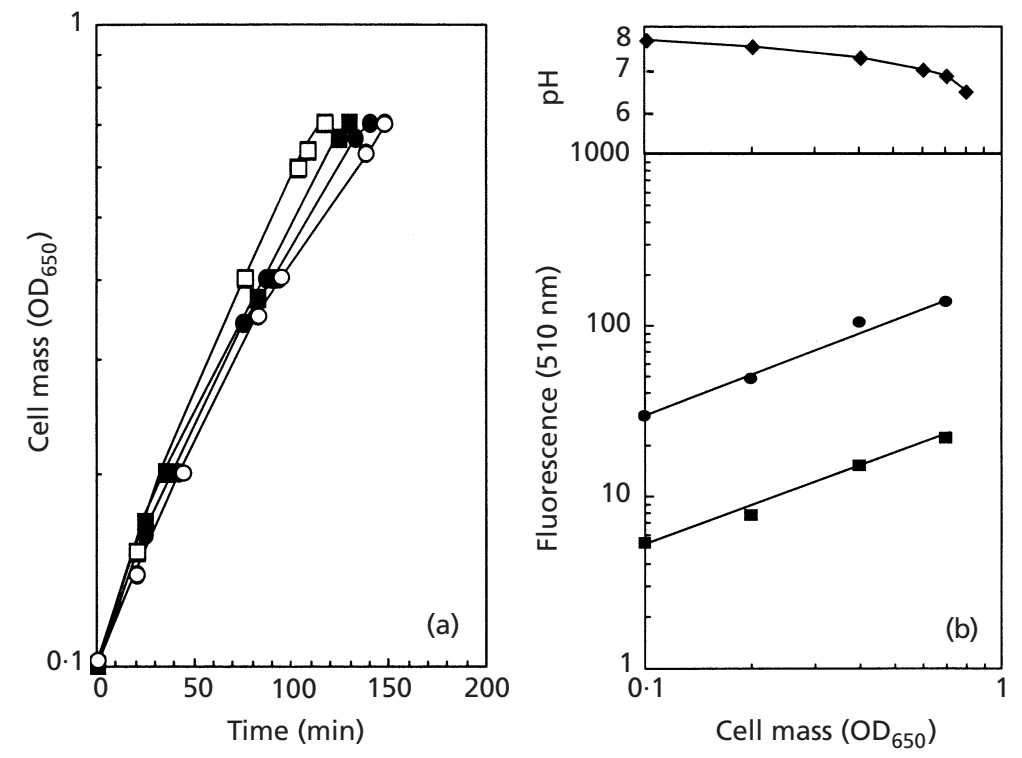

Fig. 2. Influence of GFP synthesis on growth (a) and fluorescence in cuvettes (b) of pneumococcal cells. Both parameters were measured on cultures grown in medium containing maltose and sucrose. Strains used were Rc19 ( $\square$ ), Rc19malM::gfp cat (ם), Rc19/pLS70 (O) and Rc19/pLS70GFP (•). The fluorescence due to GFP in strains Rc19/pLS70GFP and Rc19malM::gfp cat was plotted after subtraction of the fluorescence background detected in Rc19/pLS70 and Rc19, respectively. The $\mathrm{pH}$ of the medium during growth of Rc19/pLS70GFP was monitored (shown at the top of b).

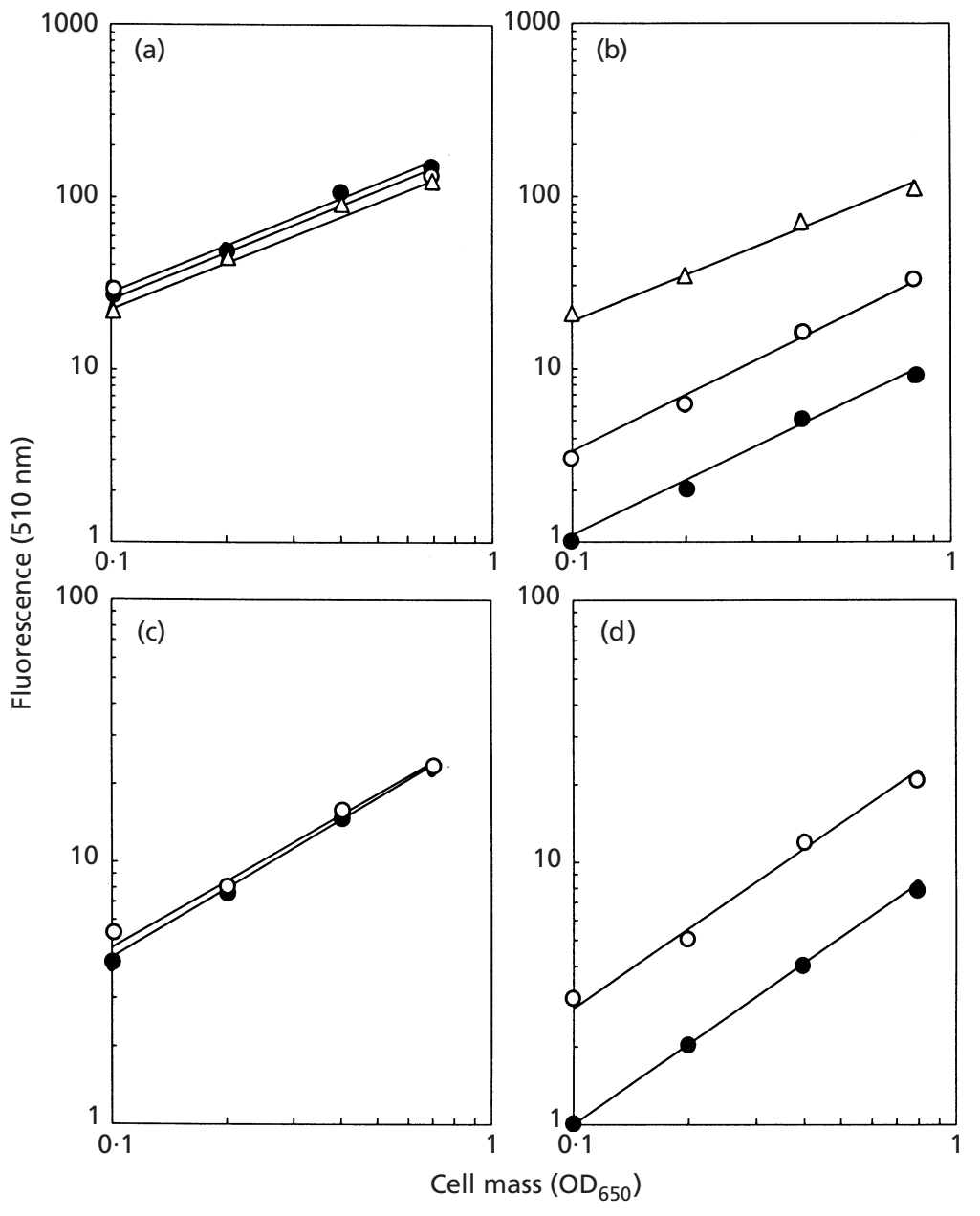

Fig. 3. Expression of GFP as a function of cell density on inducible and constitutive strains. Cultures were grown in media containing sucrose (๑), sucrose and maltose $(\bigcirc)$ or maltose $(\triangle)$. Strains used were Rc19/pLS70GFP (a), R61/pLS70GFP (b), Rc19malM::gfp cat (c) and R61malM::gfp cat (d). Fluorescence due to GFP was calculated as in Fig. 2, and the corrected values were plotted against the cell mass of the cultures.

appropriateness of the strains harbouring single or multiple copies of $g f p$, we tested whether a possible correlation between biomass and expression of GFP existed when cells were grown in media containing sucrose, maltose or a mixture of both sugars. Since amylomaltase is required for utilization of maltose as a 

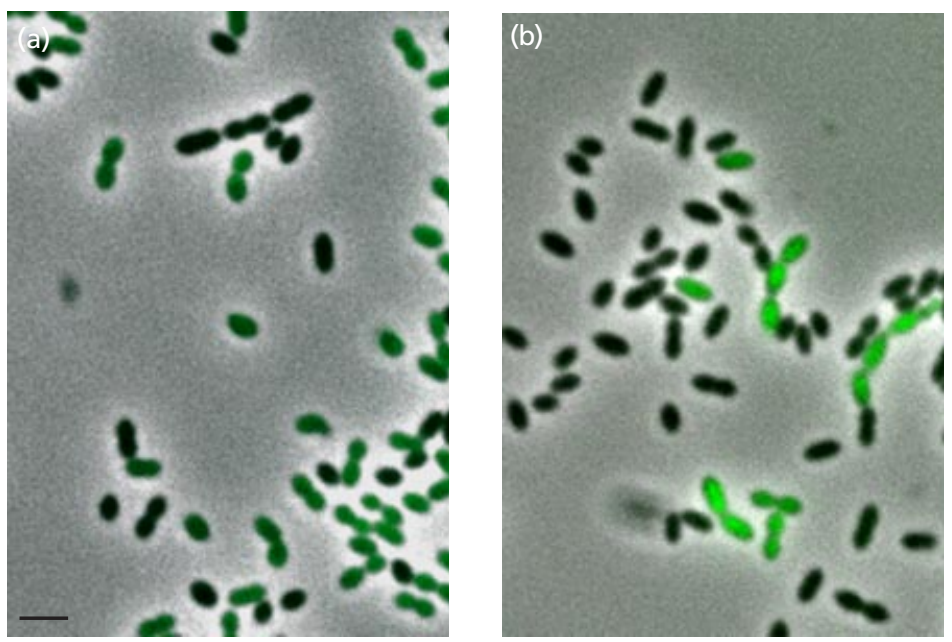

Fig. 4. Detection of expression of GFP in pneumococcal cells by fluorescence microscopy. Mixed cultures of exponentially growing cells of Rc19 and of Rc19malM::gfp cat (a) or of Rc19/pLS70GFP and Rc19/pLS70 (b) were analysed. Bar, $1 \mu \mathrm{m}$.

carbon source by pneumococci, strains harbouring the malM:: gfp cat insertion could not be grown in medium containing only maltose. Growth of the cultures was monitored by spectrophotometry, and the levels of GFP were measured by fluorescence spectroscopy in growth medium at different stages of cell growth (Fig. 3). In the case of strains Rc19 harbouring $g f p$, the fluorescence of the cultures increased with cell mass, independently of the carbon source (Fig. 3a, c), as expected for a constitutive strain. There was a clear relationship between the levels of GFP synthesized and the dosage of the gfp gene, GFP being about 6-7 times higher in Rc19/pLS70GFP than in the strain harbouring one chromosomal copy of $g f p$. These differences agree with previous measurements of amylomaltase activity when tested in a single chromosomal copy or in plasmid pLS70 (Stassi et al., 1982). Consequently, the defective MalR synthesized by Rc19 does not repress promoter $P_{\mathrm{M}}$ and $g f p$ expression is constitutive.

In the case of strain R61 (malR-proficient) harbouring pLS70GFP, partial or total de-repression of GFP synthesis was found in medium containing maltose and sucrose or only maltose, respectively (Fig. 3b). In the medium containing both sugars, higher levels of GFP were detected when the concentration of maltose was increased, until the values measured were the same as those obtained for cultures grown only in maltose (not shown). When the $g f p$ gene was provided in a single chromosomal copy (Fig. 3d), a similar result was found for cultures grown in sucrose plus maltose. In these cases, the GFP levels found in maltose-grown cultures were comparable to those obtained in the isogenic Rc19 strains (compare Fig. 3a with $3 \mathrm{~b}$, and $3 \mathrm{c}$ with $3 \mathrm{~d}$ ), and are consistent with the amylomaltase levels previously measured (Stassi et al., 1982). However, when the cultures were grown in sucrose (Figs $3 \mathrm{~b}, \mathrm{~d}$ ), low but significant amounts of GFP were detectable, indicating that repression by MalR was not enough to shut off $g f p$ transcription from promoter $P_{\mathrm{M}}$. This would be expected when $g f p$ was present in multiple copies but not when a single copy was assayed, a result for which we have no explanation at present. We conclude that over the range of growth analysed, the expression of GFP can be reliably quantified and, in consequence, GFP can be used to monitor pneumococcal cells.

\section{Detection of GFP-expressing pneumococcal cells by fluorescence microscopy}

To test whether S. pneumoniae Rc19 cells harbouring single or multiple copies of $g f p$ could be visualized by fluorescence microscopy, the strains were grown and directly analysed in growth medium (without fixing) by phase-contrast or by fluorescence microscopy. The analysis was also performed in mixed cultures of Rc19 expressing or not expressing GFP. All cells harbouring a single $g f p$ copy or pLS70GFP were fluorescently labelled (not shown). Employment of mixed cultures of cells expressing or not expressing GFP allowed us to distinguish the two isogenic strains, and expression of GFP in monocopy (Fig. 4a) or multicopy (Fig. 4b) did not affect cell morphology. We believe that this is a fundamental step toward monitoring the adherence of $S$. pneumoniae to human cells, since one copy of the $g f p$ gene, integrated into the bacterial chromosome, is enough to follow their fate upon invasion. Adhesion can be thus monitored directly without the need for tedious analyses of binding of $S$. pneumoniae to human cell lines.

\section{ACKNOWLEDGEMENTS}

Thanks are due to V. de Lorenzo for providing us with pGreenTIR and to S. A. Lacks for a gift of the pneumococcal strain Rc19. This research was financed by Comisión Interministerial de Ciencia y Tecnología, grant BIO97-0347 (to M.E.), by Comunidad Autónoma de Madrid, grants 08.3 / 0028.1/98 (to P.L.) and 07B/0030/1999 (to M.E.), and by the European Union, grant BIO4-CT98-0424 EU (to P.L.).

\section{REFERENCES}

Ballester, S., Lopez, P., Espinosa, M., Alonso, J. C. \& Lacks, S. A. (1989). Plasmid structural instability associated with pC194 replication functions. J Bacteriol 171, 2271-2277. 
Ballester, S., Alonso, J. C., López, P. \& Espinosa, M. (1990). Comparative expression of the pC194 cat gene in Streptococcus pneumoniae, Bacillus subtilis and Escherichia coli. Gene 86, 71-79.

Burdett, V. (1980). Identification of tetracycline-resistant Rplasmids in Streptococcus agalactiae (group B). Antimicrob Agents Chemother 18, 753-760.

Christensen, B. B., Sternberg, C. \& Molin, S. (1996). Bacterial plasmid conjugation on semi-solid surfaces monitored with the green fluorescent protein (GFP) from Aequorea victoria as a marker. Gene 173, 59-65.

Fernández de Palencia, P., Nieto, C., Acebo, P., Espinosa, M. \& López, P. (2000). Expression of green fluorescent protein in Lactococcus lactis. FEMS Microbiol Lett 183, 229-234.

Geoffroy, M. C., Guyard, C., Quatannens, B., Pavan, S., Lange, M. \& Mercenier, A. (2000). Use of green fluorescent protein to tag lactic acid bacterium strains under development as live vaccine vectors. Appl Environ Microbiol 66, 383-391.

Horinouchi, S. \& Weisblum, B. (1982). Nucleotide sequence and functional map of pC194, a plasmid that specifies inducible chloramphenicol resistance. J Bacteriol 150, 815-825.

Lacks, S. A. (1968). Genetic regulation of maltosaccharide utilization in Pneumococcus. Genetics 60, 685-706.

Lacks, S. A., Lopez, P., Greenberg, B. \& Espinosa, M. (1986). Identification and analysis of genes for tetracycline resistance and replication functions in the broad-host-range plasmid pLS1. $J$ Mol Biol 192, 753-765.

Lemon, K. P. \& Grossman, A. D. (1998). Localization of bacterial DNA polymerase: evidence for a factory model of replication. Science 282, 1516-1519.

Lewis, P. J. \& Errington, J. (1996). Use of green fluorescent protein for detection of cell-specific gene expression and subcellular protein localization during sporulation in Bacillus subtilis. Microbiology 142, 733-740.

López, P., Espinosa, M., Stassi, D. L. \& Lacks, S. A. (1982). Facilitation of plasmid transfer in Streptococcus pneumoniae by chromosomal homology. J Bacteriol 150, 692-701.

Miller, W. G. \& Lindow, S. E. (1997). An improved GFP cloning cassette designed for prokaryotic transcriptional fusions. Gene 191, 149-153.

Nieto, C., Espinosa, M. \& Puyet, A. (1997). The maltose/ maltodextrin regulon of Streptococcus pneumoniae. Differential promoter regulation by the transcriptional repressor MalR. J Biol Chem 272, 30860-30865.

Puyet, A. \& Espinosa, M. (1993). Structure of the maltodextrinuptake locus of Streptococcus pneumoniae. Correlation to the Escherichia coli maltose regulon. J Mol Biol 230, 800-811.

Puyet, A., Ibañez, A. M. \& Espinosa, M. (1993). Characterization of the Streptococcus pneumoniae maltosaccharide regulator MalR, a member of the LacI-GalR family of repressors displaying distinctive genetic features. J Biol Chem 268, 25402-25408.

Sambrook, J., Fritsch, E. F. \& Maniatis, T. (1989). Molecular Cloning: a Laboratory Manual, 2nd edn. Cold Spring Harbor, NY: Cold Spring Harbor Laboratory.

Scott, K. P., Mercer, D. K., Richardson, A. J., Melville, C. M., Glover, L. M. \& Flint, H. J. (2000). Chromosomal integration of the green fluorescent protein gene in lactic acid bacteria and the survival of marked strains in human gut simulations. FEMS Microbiol Lett 182, 23-27.

del Solar, G., Diaz, R. \& Espinosa, M. (1987). Replication of the streptococcal plasmid pMV158 and derivatives in cell-free extracts of Escherichia coli. Mol Gen Genet 206, 428-435.

del Solar, G., Giraldo, R., Ruiz-Echevarria, M. J., Espinosa, M. \& Diaz-Orejas, R. (1998). Replication and control of circular bacterial plasmids. Microbiol Mol Biol Rev 62, 434-464.

Somkuti, G. A. \& Steinberg, D. H. (1999). Promoter activity of the pER341-borne ST(Phsp) in heterologous gene expression in Escherichia coli and Streptococcus thermophilus. FEMS Microbiol Lett 179, 431-436.

Stassi, D. L., Lopez, P., Espinosa, M. \& Lacks, S. A. (1982). Cloning of chromosomal genes in Streptococcus pneumoniae. Proc Natl Acad Sci USA 78, 7028-7032.

Sullivan, K. F. \& Kay, S. A. (1999). Green Fluorescent Protein. San Diego: Academic Press.

Received 1 December 1999; revised 21 February 2000; accepted 20 March 2000. 